\title{
Photoelectron Sheath near the Lunar Surface: Fully Kinetic Modeling and Uncertainty Quantification Analysis
}

\author{
Jianxun Zhao*, Xinpeng $\mathrm{Wei}^{\dagger}$, Zhangli Hu${ }^{\ddagger}$, Xiaoming $\mathrm{He}^{\S}$, and Daoru Han ${ }^{\mathbb{I}}$ \\ Missouri University of Science and Technology, Rolla, Missouri 65409 \\ Zhen $\mathrm{Hu}$ ll \\ University of Michigan - Dearborn, Dearborn, Michigan 48128
}

Xiaoping $\mathrm{Du}^{* *}$

Indiana University - Purdue University Indianapolis, Indianapolis, Indiana 46202

\begin{abstract}
This paper presents a modeling and uncertainty quantification (UQ) study of the photoelectron sheath near the lunar surface. A fully kinetic 3-D finite-difference (FD) particle-in-cell (PIC) code is utilized to simulate the plasma interaction near the lunar surface and the resulting photoelectron sheath. For the uncertainty quantification analysis, this FD-PIC code is treated as a black box providing high-fidelity quantities of interest, which are also used to construct efficient reduced-order models to perform UQ analysis. 1-D configuration is chosen to present the analytic sheath solution as well as to demonstrate the procedure and capability of the UQ analysis.
\end{abstract}

\section{Nomenclature}

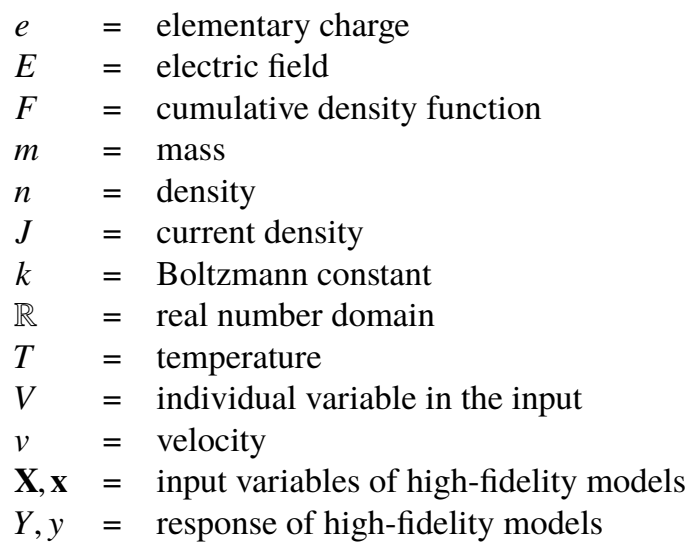

Greek letters

$\phi=$ electric potential

$\Omega=$ computation domain

Subscript

c $=$ captured

\footnotetext{
*Graduate Research Assistant, Department of Mechanical and Aerospace Engineering, 400 W. 13th St., Rolla, MO 65409, AIAA Student Member.

${ }^{\dagger}$ Graduate Research Assistant, Department of Mechanical and Aerospace Engineering. 400 W. 13th St., Rolla, MO 65409.

${ }^{\ddagger}$ Graduate Research Assistant, Department of Mechanical and Aerospace Engineering. 400 W. 13th St., Rolla, MO 65409. Currently at Caterpillar Inc., 14009 Old Galena Rd, Mossville, IL 61552.

$\S$ Associate Professor, Department of Mathematics and Statistics. 400 W. 12th St., Rolla, MO 65409.

"I Assistant Professor, Department of Mechanical and Aerospace Engineering, 400 W. 13th St., Rolla, MO 65409, AIAA Member. handao@mst . edu

"Assistant Professor, Department of Industrial and Manufacturing Systems Engineering, 2250 HPEC, Dearborn, MI 48128, AIAA Member.

***Professor, Purdue School of Engineering and Technology, 799 W. Michigan St., Indianapolis, IN 46202.
} 


$\begin{aligned} \mathrm{d} & =\text { drifting } \\ \mathrm{f} & =\text { free } \\ \mathrm{m} & =\text { minimum } \\ \mathrm{r} & =\text { reflected } \\ \mathrm{s} & =\text { surface } \\ \mathrm{th} & =\text { thermal } \\ p & =\text { photoelectron } \\ e & =\text { electron } \\ i & =\text { ion }\end{aligned}$

\section{Introduction}

$\mathrm{T}$ HIS paper considers plasma charging on the lunar surface with a focus on photoelectron sheath. The plasma species includes ambient solar wind (protons and electrons) and photoelectrons emitted from the illuminated lunar surface. This work is motivated by the high computational cost associated with uncertainty quantification (UQ) analysis of plasma simulations using high-fidelity fully kinetic models. In this paper, we study the photoelectron sheath near the lunar surface with a focus on effects of variables of uncertainty (such as the ambient electron density or photoelectron temperature) on the plasma environment. A fully kinetic 3-D finite-difference (FD) particle-in-cell (PIC) code is utilized to simulate the plasma interaction near the lunar surface and the resulting photoelectron sheath. For the uncertainty quantification analysis, this PIC code is treated as a black box providing high-fidelity quantities of interest, which are also used to construct efficient reduced-order models to perform UQ analysis. A 1-D configuration is first studied to demonstrate the procedure and capability of the UQ analysis. The rest of the paper is organized as follows. Section III presents the analytic and numerical solutions of the 1-D photoelectron sheath. Verification and validation of the FD-PIC code for photoelectron sheath solution is shown. Section IV]describes the Kriging model and the uncertainty quantification approach. Section V discusses the UQ analysis of the 1-D photoelectron sheath. The conclusion is given in SectionVI

\section{Analytic and Numerical Solutions of 1-D Photoelectron Sheath}

\section{A. Analytic Solution}

\section{Sheath Model}

The essential of the work in this research is following [1] and [2], but is extended to include the drifting velocity of solar wind electrons in analytic calculation, and compare the result of a numerical simulation with the analytic solution.

According to [1] and [2], there will be 3 types of electric potential profile in the photoelectron sheath: Sheath Type A, Sheath Type B, and Sheath Type C, as shown in Fig. 1. For each sheath type, there will be different populations of electrons. The solar wind electron contains "free solar wind electrons", representing electrons which are able to reach the lunar surface, and "reflected electrons", representing electrons which are reflected by the potential barrier. The photoelectron contains "free photoelectrons", which are able to reach the infinity, and "captured photoelectrons", which are attracted back to the lunar surface. The reflected and captured electrons are both consisted as two equal parts but traveling in opposite directions, as shown in Fig. 2

In these three sheath profiles, Type A is a non-monotonic potential profile, the curve of Type A will first decrease from the surface to the minimum potential, then it will increase to the potential at infinity, which is zero. Type B will decrease from the surface potential to the potential at infinity. Type $\mathrm{C}$ will increase from the surface potential to the potential at infinity. Both Type B and Type $\mathrm{C}$ are monotonic. The profiles are shown in Fig. 3.

\section{Electron Distribution}

There are two kinds of electrons in the computation domain. The solar wind electrons, which is originating from the ambient plasma, and the photoelectrons, which is originating from the lunar surface. In this computation, we assume that both kinds of electrons follow the Maxwellian distribution in the photoelectron sheath, the distribution function is given in Eq. (1). 


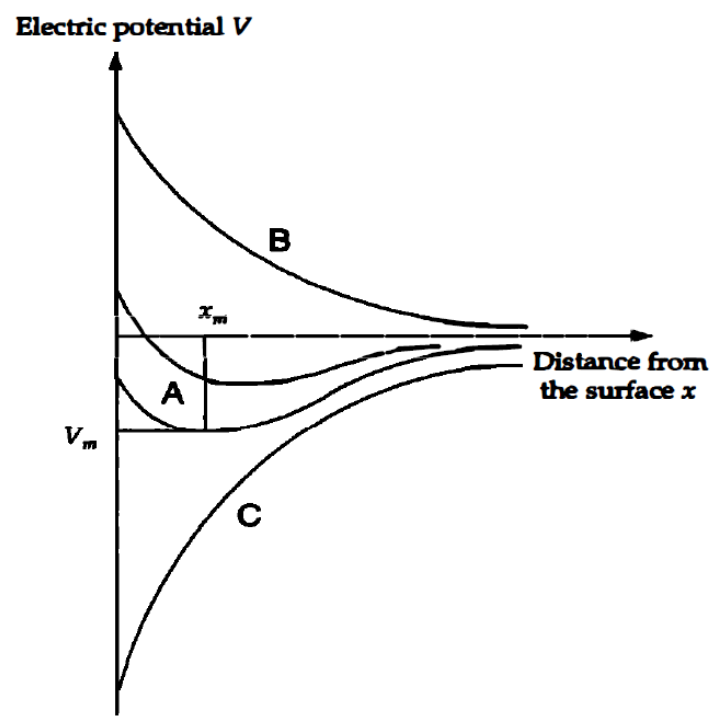

Fig. 1 Assumed potential profiles as a function of the distance from the surface [2]

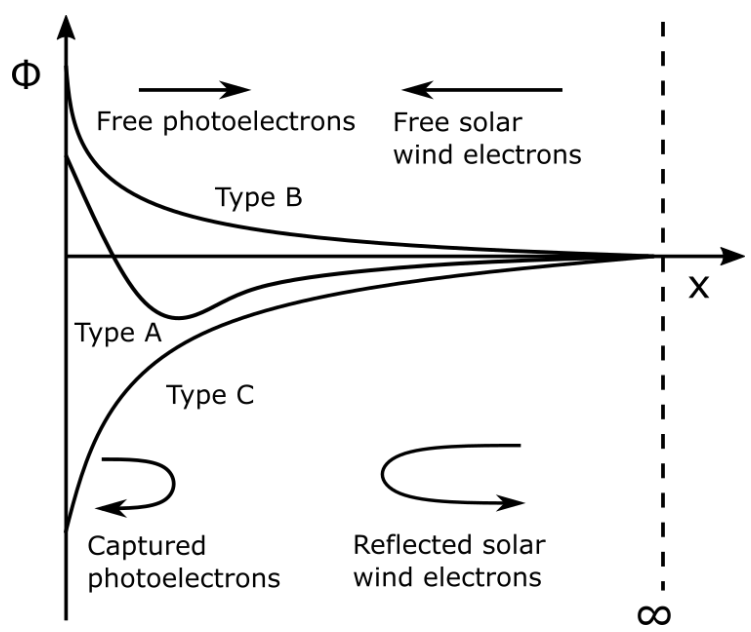

Fig. 2 Different populations of electrons as described in [2]

$$
f(v)=\frac{1}{\sqrt{\pi} v_{\mathrm{th}}} \exp \left(-\frac{v^{2}}{v_{\mathrm{th}}^{2}}\right)
$$

where the $v_{\text {th }}=\sqrt{\frac{2 k T}{m}}$ is the thermal velocity of the particle.

Equation (1) is for stationary Maxwellian distribution, for drifting Maxwellian distribution, the equation will be written as

$$
f(v)=\frac{1}{\sqrt{\pi} v_{\mathrm{th}}} \exp \left[-\frac{\left(v-v_{\mathrm{d}}\right)^{2}}{v_{\mathrm{th}}^{2}}\right]
$$

where $v_{\mathrm{d}}$ is the drifting velocity.

\section{Densities}

The density of each population of electrons is given as 


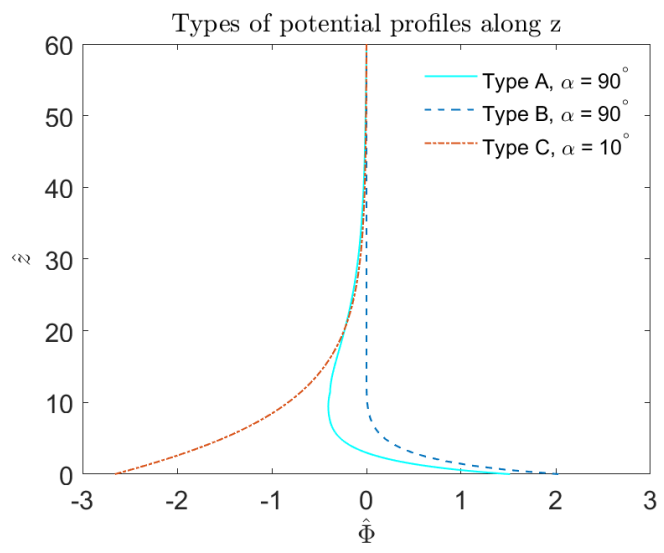

Fig. 3 Potential profiles obtained from the analytic solution with normalized coordinates and potential

$$
\begin{aligned}
& n_{e, \mathrm{f}}(z)=\int_{v_{\mathrm{m}}}^{\infty} n_{e, \infty} f(v) \mathrm{d} v \\
& =\int_{v_{\mathrm{m}}}^{\infty} n_{e, \infty} \frac{1}{\sqrt{\pi} v_{e, \mathrm{th}}} \exp \left[-\frac{\left(v-v_{\mathrm{d}}\right)^{2}}{v_{e, \mathrm{th}^{2}}}\right] \exp \left[\frac{e\left(\phi_{(z)}-\phi_{\infty}\right)}{k T_{e}}\right] \mathrm{d} v \\
& =\frac{n_{e, \infty}}{2} \exp \left[\frac{e\left(\phi_{(z)}-\phi_{\infty}\right)}{k T_{e}}\right]\left[1-\operatorname{erf}\left(\sqrt{\frac{e\left(\phi_{(z)}-\phi_{\mathrm{m}}\right)}{k T_{e}}}-\frac{v_{\mathrm{d}}}{v_{e, \mathrm{th}}}\right)\right] \\
& n_{p, \mathrm{f}}(z)=\int_{v_{\mathrm{m}}}^{\infty} n_{p, 0} f(v) \mathrm{d} v \\
& =\int_{v_{\mathrm{m}}}^{\infty} n_{p, 0} \frac{1}{\sqrt{\pi} v_{p, \mathrm{th}}} \exp \left[-\frac{\left(v-v_{\mathrm{d}}\right)^{2}}{v_{p, \mathrm{th}^{2}}}\right] \exp \left[\frac{e\left(\phi_{(z)}-\phi_{0}\right)}{k T_{p}}\right] \mathrm{d} v \\
& =\frac{n_{p, 0}}{2} \exp \left[\frac{e\left(\phi_{(z)}-\phi_{0}\right)}{k T_{p}}\right]\left[1-\operatorname{erf}\left(\sqrt{\frac{e\left(\phi_{(z)}-\phi_{\mathrm{m}}\right)}{k T_{p}}}\right)\right] \\
& n_{e, \mathrm{r}}(z)=2 \int_{0}^{v_{\mathrm{m}}} n_{e, \infty} f(v) \mathrm{d} v \\
& =2 \int_{0}^{v_{\mathrm{m}}} n_{e, \infty} \frac{1}{\sqrt{\pi} v_{e, \mathrm{th}}} \exp \left[-\frac{\left(v-v_{\mathrm{d}}\right)^{2}}{v_{e, \mathrm{th}^{2}}}\right] \exp \left[\frac{e\left(\phi_{(z)}-\phi_{\infty}\right)}{k T_{e}}\right] \mathrm{d} v \\
& =n_{e, \infty} \exp \left[\frac{e\left(\phi_{(z)}-\phi_{\infty}\right)}{k T_{e}}\right]\left[\operatorname{erf}\left(\sqrt{\frac{e\left(\phi_{(z)}-\phi_{\mathrm{m}}\right)}{k T_{e}}}-\frac{v_{\mathrm{d}}}{v_{e, \mathrm{th}}}\right)+\operatorname{erf}\left(\frac{v_{\mathrm{d}}}{v_{e, \mathrm{th}}}\right)\right] \\
& n_{p, \mathrm{c}}(z)=2 \int_{0}^{v_{\mathrm{m}}} n_{p, 0} f(v) \mathrm{d} v \\
& =2 \int_{0}^{v_{\mathrm{m}}} n_{p, 0} \frac{1}{\sqrt{\pi} v_{p, \mathrm{th}}} \exp \left[-\frac{v^{2}}{v_{p, \mathrm{th}^{2}}}\right] \exp \left[\frac{e\left(\phi_{(z)}-\phi_{0}\right)}{k T_{p}}\right] \mathrm{d} v \\
& =n_{p, 0} \exp \left[\frac{e\left(\phi_{(z)}-\phi_{0}\right)}{k T_{p}}\right] \operatorname{erf}\left(\sqrt{\frac{e\left(\phi_{(z)}-\phi_{\mathrm{m}}\right)}{k T_{p}}}\right)
\end{aligned}
$$

where $n_{e, \mathrm{f}}, n_{p, \mathrm{f}}, n_{e, \mathrm{r}}$, and $n_{p, \mathrm{c}}$ represents the density of free solar wind electrons, free photoelectrons, reflected solar wind electrons, and captured photoelectrons, respectively.

$n_{e, \infty}$ and $v_{e, \text { th }}$ is the density at infinity and thermal velocity of solar wind electrons, $n_{p, 0}$ and $v_{p, \text { th }}$ is the density 
at surface and thermal velocity of photoelectrons. $v_{\mathrm{m}}=\sqrt{\frac{2 e\left(\phi_{(z)}-\phi_{\mathrm{m}}\right)}{m_{e}}}$ is the minimum velocity that required for the electrons to travel over the potential barrier. $\phi_{\mathrm{m}}$ is the minimum potential, and $\phi_{\infty}$ is the potential at infinity.

The ion density is obtained as

$$
n_{i}(z)=\frac{n_{i, \infty} v_{i, \infty}}{v_{i}(z)}=n_{i, \infty}\left(1-\frac{2 e \phi_{(z)}}{v_{i, \infty}^{2} m_{i}}\right)^{-\frac{1}{2}}
$$

where $n_{i, \infty}$ and $v_{i, \infty}$ is the density and velocity of solar wind ions at infinity.

Then the Poisson's equation can be written as

$$
\frac{\mathrm{d}^{2} \phi}{\mathrm{d} z^{2}}=-\frac{e}{\epsilon_{0}}\left(n_{i}-n_{e, \mathrm{f}}-n_{e, \mathrm{r}}-n_{p, \mathrm{f}}-n_{p, \mathrm{c}}\right)
$$

\section{Charge Neutrality}

We assume the total density at infinity is neutral in this computation, which can be represented in the equation as

$$
n_{e}(\infty)+n_{p}(\infty)-n_{i}(\infty)=n_{e, \mathrm{f}}(\infty)+n_{e, \mathrm{r}}(\infty)+n_{p, \mathrm{f}}(\infty)-n_{i}(\infty)=0
$$

Equation (9) can also be written as

$$
\frac{n_{e, \infty}}{2}\left[1+2 \operatorname{erf}\left(\frac{v_{\mathrm{d}}}{v_{e, \mathrm{th}}}\right)+\operatorname{erf}\left(\sqrt{-\frac{e \phi_{\mathrm{m}}}{k T_{e}}}-\frac{v_{\mathrm{d}}}{v_{e, \mathrm{th}}}\right)\right]+\frac{n_{p, 0}}{2} \exp \left(-\frac{e \phi_{0}}{k T_{p}}\right)\left(1-\operatorname{erf} \sqrt{-\frac{e \phi_{\mathrm{m}}}{k T_{p}}}\right)-n_{i, \infty}=0
$$

\section{Current Density}

According to the assumption, there will be no net current flow at infinity when the situation reaches the steady state. The zero current density at infinity can be obtained as

$$
J_{e, \mathrm{f}}(\infty)+J_{p, \mathrm{f}}(0)-J_{i}(\infty)=n_{e, \mathrm{f}} \int_{v_{\mathrm{m}}(0)}^{\infty} v f_{p}(v) \mathrm{d} v+n_{p, \mathrm{f}} \int_{-\infty}^{-v_{\mathrm{m}}(\infty)} v f_{e}(v) \mathrm{d} v+n_{i, \infty} v_{i, \infty}=0
$$

The reflected solar wind electrons and the captured photoelectrons are two equal parts that are traveling in opposite directions, so they will not contribute to the current.

Then Eq. (11) can be written as

$$
\begin{array}{r}
n_{p, 0} \exp \left[\frac{e\left(\phi_{\mathrm{m}}-\phi_{0}\right)}{k T_{p}}\right]-n_{e, \infty} \sqrt{\frac{T_{e}}{T_{p}}}\left\{\exp \left[-\left(\sqrt{-\frac{e \phi_{\mathrm{m}}}{k T_{e}}}-\frac{v_{\mathrm{d}}}{v_{e, \mathrm{th}}}\right)^{2}\right]\right. \\
\left.+\frac{v_{\mathrm{d}}}{v_{p, \mathrm{th}}} \sqrt{\pi} \operatorname{erfc}\left(\sqrt{-\frac{e \phi_{\mathrm{m}}}{k T_{e}}}-\frac{v_{\mathrm{d}}}{v_{e, \mathrm{th}}}\right)\right\}+n_{i, \infty} \sqrt{2 \pi \frac{T_{e}}{T_{p}} \frac{m_{e}}{m_{i}}} M=0
\end{array}
$$

\section{Electric Field}

To obtain the analytic solution of Type A, we need to calculate the minimum potential, then the electric field at infinity need to be introduced.

The electric field of each species is obtained as 


$$
\begin{aligned}
& E_{e, \mathrm{f}}=2 \int_{\phi_{\mathrm{m}}}^{\phi_{(z)}} n_{e, \mathrm{f}} d \phi \\
& =\frac{T_{e} n_{e, \infty}}{T_{p} n_{p}}\left\{\exp \left[\frac{e\left(\phi_{(z)}-\phi_{\infty}\right)}{k T_{e}}\right]\left[1-\operatorname{erf}\left(\sqrt{\frac{e\left(\phi_{(z)}-\phi_{\mathrm{m}}\right)}{k T_{e}}}-\frac{v_{\mathrm{d}}}{v_{e, \mathrm{th}}}\right)\right]-\exp \left[\frac{e\left(\phi_{\mathrm{m}}-\phi_{\infty}\right)}{k T_{e}}\right]\right. \\
& \left.\left[1-\operatorname{erf}\left(-\frac{v_{\mathrm{d}}}{v_{e, \mathrm{th}}}\right)\right]+\frac{v_{e, \mathrm{th}}}{\sqrt{\pi} v_{\mathrm{d}}} \exp \left[\frac{e\left(\phi_{\mathrm{m}}-\phi_{\infty}\right)}{k T_{e}}-\frac{v_{\mathrm{d}}^{2}}{v_{e, \mathrm{th}^{2}}^{2}}\right]\left[\exp \left(2 \frac{v_{\mathrm{d}}}{v_{e, \mathrm{th}}} \sqrt{\frac{e\left(\phi_{(z)}-\phi_{\mathrm{m}}\right)}{k T_{e}}}\right)-1\right]\right\} \\
& E_{p, \mathrm{f}}=2 \int_{\phi_{\mathrm{m}}}^{\phi_{(z)}} n_{p, \mathrm{f}} d \phi \\
& =\frac{n_{p, 0}}{n_{p}}\left\{\exp \left[\frac{e\left(\phi_{(z)}-\phi_{0}\right)}{k T_{p}}\right]\left[1-\operatorname{erf}\left(\sqrt{\frac{e\left(\phi_{(z)}-\phi_{\mathrm{m}}\right)}{k T_{p}}}\right)\right]\right. \\
& \left.-\exp \left[\frac{e\left(\phi_{\mathrm{m}}-\phi_{0}\right)}{k T_{p}}\right]\left[1-\frac{2}{\sqrt{\pi}} \sqrt{\frac{e\left(\phi_{(z)}-\phi_{\mathrm{m}}\right)}{k T_{p}}}\right]\right\} \\
& E_{e, \mathrm{r}}=2 \int_{\phi_{\mathrm{m}}}^{\phi_{(z)}} n_{e, \mathrm{f}} d \phi \\
& =\frac{2 T_{e} n_{e, \infty}}{T_{p} n_{p}}\left\{\exp \left[\frac{e\left(\phi_{(z)}-\phi_{\infty}\right)}{k T_{e}}\right]\left[\operatorname{erf}\left(\sqrt{\frac{e\left(\phi_{(z)}-\phi_{\mathrm{m}}\right)}{k T_{e}}}-\frac{v_{\mathrm{d}}}{v_{e, \mathrm{th}}}\right)+\operatorname{erf}\left(\frac{v_{\mathrm{d}}}{v_{e, \mathrm{th}}}\right)\right]\right. \\
& \left.-\frac{v_{e, \mathrm{th}}}{\sqrt{\pi} v_{\mathrm{d}}} \exp \left[\frac{e\left(\phi_{\mathrm{m}}-\phi_{\infty}\right)}{k T_{e}}-\frac{v_{\mathrm{d}}^{2}}{v_{e, \mathrm{th}}^{2}}\right]\left[\exp \left(2 \frac{v_{\mathrm{d}}}{v_{e, \mathrm{th}}} \sqrt{\frac{e\left(\phi_{(z)}-\phi_{\mathrm{m}}\right)}{k T_{e}}}\right)-1\right]\right\} \\
& E_{p, \mathrm{c}}=2 \int_{\phi_{\mathrm{m}}}^{\phi_{(z)}} n_{p, \mathrm{c}} d \phi \\
& =\frac{2 n_{p, 0}}{n_{p}}\left\{\exp \left[\frac{e\left(\phi_{(z)}-\phi_{0}\right)}{k T_{p}}\right]\left[\operatorname{erf}\left(\sqrt{\frac{e\left(\phi_{(z)}-\phi_{\mathrm{m}}\right)}{k T_{p}}}\right)\right]\right. \\
& \left.-\frac{2}{\sqrt{\pi}} \exp \left[\frac{e\left(\phi_{\mathrm{m}}-\phi_{0}\right)}{k T_{p}}\right] \sqrt{\frac{e\left(\phi_{(z)}-\phi_{\mathrm{m}}\right)}{k T_{p}}}\right\} \\
& E_{i}=-2 \int_{\phi_{\mathrm{m}}}^{\phi_{(z)}} n_{i} d \phi=\frac{2 T_{e} n_{i, \infty}}{T_{p} n_{p}} M^{2}\left[\sqrt{1-\frac{2 e \phi_{(z)}}{k T_{e} M^{2}}}-\sqrt{1-\frac{2 e \phi_{\mathrm{m}}}{k T_{e} M^{2}}}\right]
\end{aligned}
$$

where $E_{e, \mathrm{f}}, E_{p, \mathrm{f}}, E_{e, \mathrm{r}}, E_{p, \mathrm{c}}$, and $E_{i}$ is the electric field of free solar wind electrons, free photoelectrons, reflected solar wind electrons, captured photoelectrons, and ions, respectively. $n_{p}$ is the photoelectron density when the elevation angle is $90^{\circ}$. And $M=\frac{v_{d}}{C_{\mathrm{s}}}$ is the ion Mach number.

The zero electric field at infinity can be obtained as

$$
E(\infty)=E_{e, \mathrm{f}}(\infty)+E_{e, \mathrm{r}}(\infty)+E_{p, \mathrm{f}}(\infty)+E_{i}(\infty)=0
$$

\section{B. Verification of the FD-PIC Simulation of 1-D Photoelectron Sheath}

\section{Comparison of Analytic Solution and Simulation}

The comparison of the results of analytic solution and simulation are presented in this section. The drifting velocities of the solar wind electrons and ions are both $468 \mathrm{~km} / \mathrm{s}$, the densities at infinity are both $8.7 \mathrm{~cm}^{-3}$, and the thermal temperatures are $12 \mathrm{eV}$ and $10 \mathrm{eV}$, respectively. The parameters used in this computation are listed in Table 1 . The lunar surface is considered as a flat surface, which means the elevation angle will not change at any place on the surface. 
The numerical simulation in this study is using a FD-PIC code. In this code, the collisions between particles are neglected, hence the lunar surface charging, electric field, charged particle trajectories, and space charge are solved self-consistently.

\section{Computation Domain}

In order to simulate a 1-D photoelectron sheath, the computation domain is selected as a $2 \times 2 \times 600$ region, which is shown in Fig. 4. All PIC cells are normalized, so the length of each cubic PIC cell is $\sim 1.315 / \sqrt{\sin (\alpha)} \mathrm{m}$, where the $\alpha$ is the elevation angle. The solar wind electrons and ions are traveling into the computation domain in the $X-Y$ plane, along $-z$-direction.

There are in total 180,000 solar wind electrons and ions are preloaded in the computation domain, and another 100 particles of both species, including photoelectrons, are injected into the computation domain in each time step. The simulation will run 100,000 steps for each case, which is $\sim 900$ seconds. The wall clock time is $\sim 6$ hours for each case. The time step $n$ is normalized by $\frac{1}{\omega_{p}}$, where $\omega_{p}$ is the frequency of the photoelectron.

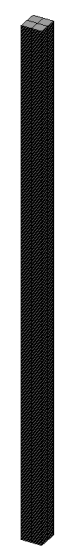

(a) The computation domain
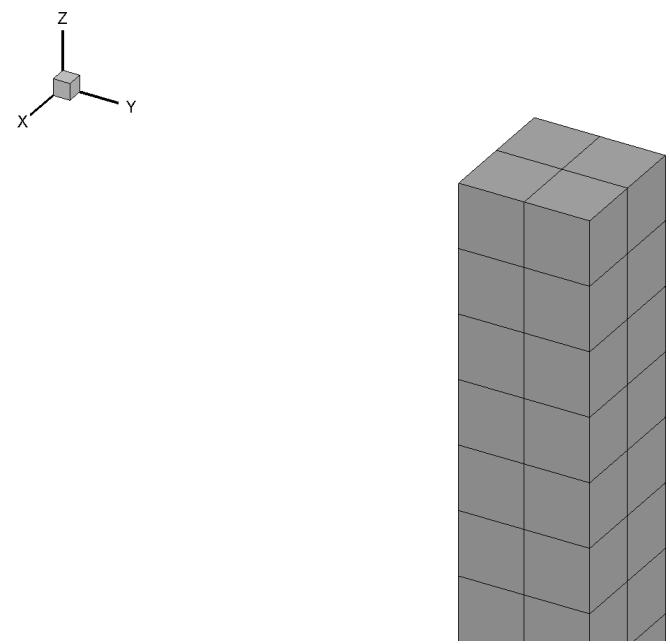

(b) Top surface of the domain

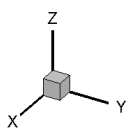

Fig. 4 The detail of the computation domain

\section{Boundary Conditions}

The zero-Dirichlet boundary condition where $\phi=0$ is applied for the $Z_{\max }$ boundary, which is treated as the reference for the electric potential. The other boundaries are all applied with the zero-Neumann boundary condition where $\frac{\partial \phi}{\partial n}=0$.

Solar wind electrons and ions are loaded in the $X-Y$ plane and injected at $Z_{\max }$, and the photoelectrons are injected at $Z_{\min }$. The boundary conditions for $y$-dimensions and $x$-dimensions are periodic and reflective, respectively. Particles are absorbed at $z$-dimensions.

\section{Results and Comparison}

Figure 5 shows the comparison of the potential profiles obtained from analytic solution and the simulation at elevation angle $\alpha=90^{\circ}$ and $\alpha=60^{\circ}$, respectively. The mesh size is $0.1 \lambda_{\mathrm{d}}$, where $\lambda_{\mathrm{d}}$ is the Debye length of the photoelectron.

When the elevation angle is large enough, the profiles of Type A and Type B can be existed at the same time in both analytic solution and the simulation. Figure 6 shows the Type B profile in the sheath at the same elevation angles as in Type A.

The reason that causes the difference between Type A and Type B is that, according to the assumption, the solar wind electron density at infinity is not equal to solar wind ion density, which is obtained by solving Eqs. (9), (11), and (18). The solution in Type A is larger than that in Type B, which will result in a larger surface potential in Type B. 


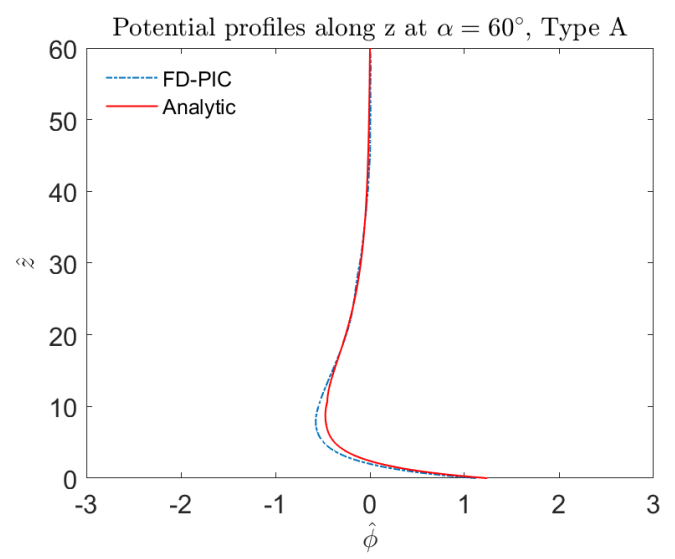

(a) $\alpha=60^{\circ}$

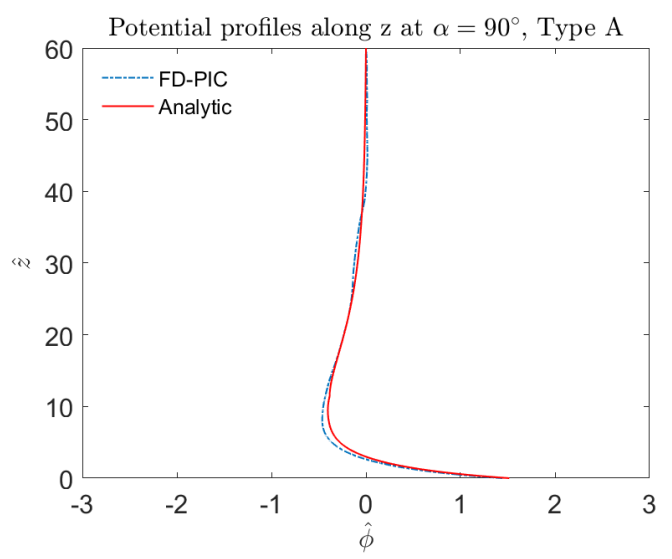

(b) $\alpha=90^{\circ}$

Fig. 5 Comparison of the Type A potential profile obtained from the analytic solution and the simulation

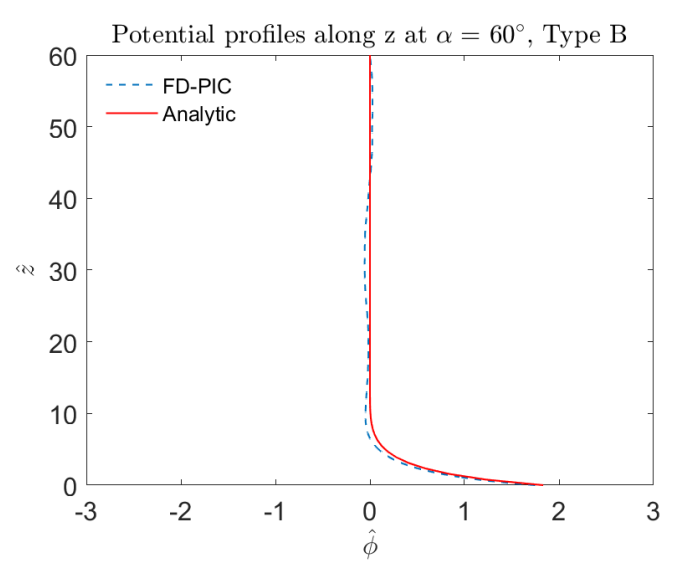

(a) $\alpha=60^{\circ}$

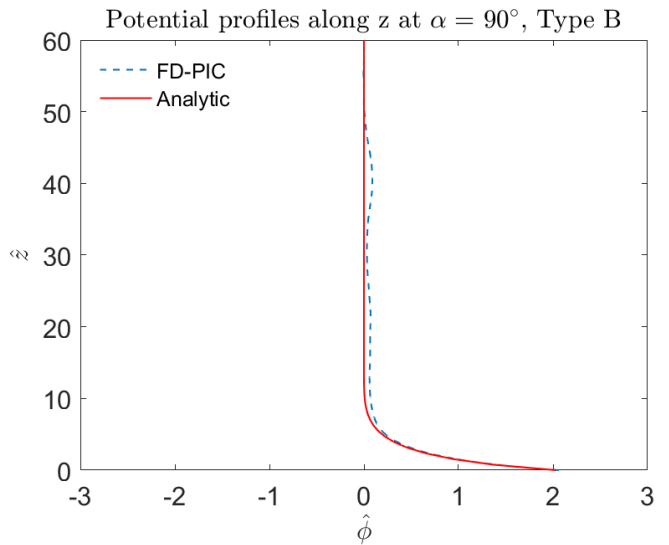

(b) $\alpha=90^{\circ}$

Fig. 6 Comparison of the Type B potential profile obtained from the analytic solution and the simulation

Figure 7 shows the comparison of the potential profiles obtained from analytic solution and the simulation at the elevation angle $\alpha=10^{\circ}$ and $\alpha=5^{\circ}$, with a drifting solar wind electrons.

\section{Surrogate Modeling and Uncertainty Quantification Approach}

\section{A. Introduction to Kriging}

Kriging [3] is based on the idea that the black-box function $g(\mathbf{x})$ is regarded as a realization of a stochastic field $G(\mathbf{x})$. First, the stochastic field is defined based on a design of experiments. Then, the Best Linear Unbiased Predictor is used to predict the output value at any input point. The model for $G(\mathbf{x})$ is given as

$$
G(\mathbf{x})=F(\mathbf{x}, \beta)+h(\mathbf{x})
$$

where $F(\mathbf{x}, \beta)$ is the deterministic part which gives an approximation of the response in mean, and $h(\mathbf{x})$ is a stationary Gaussian field with zeros mean. $F(\mathbf{x}, \beta)$ represents the trend of Kriging and corresponds to a regression model that can be written as

$$
F(\mathbf{x}, \beta)=f(\mathbf{x}) \beta
$$




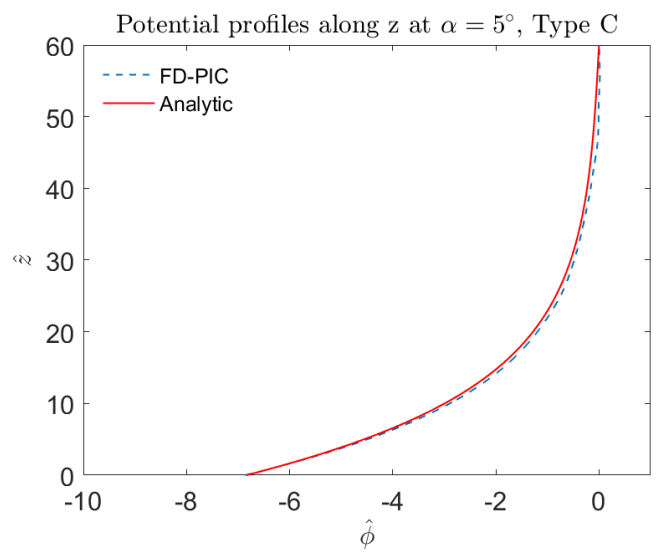

(a) $\alpha=5^{\circ}$

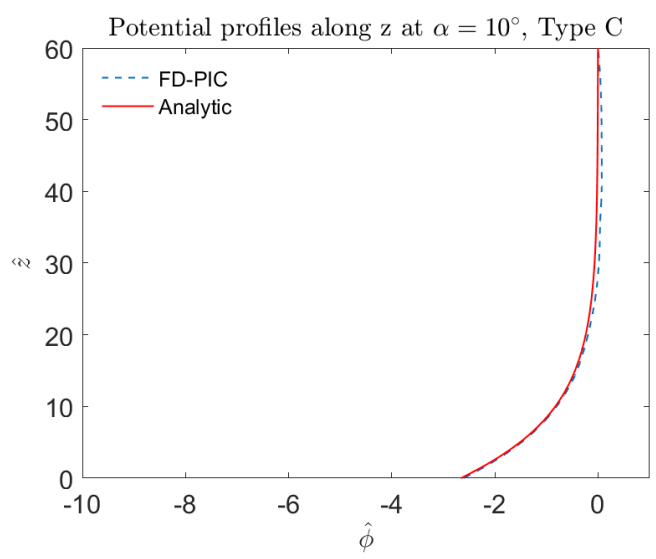

(b) $\alpha=10^{\circ}$

Fig. 7 Comparison of the Type $\mathrm{C}$ potential profile obtained from the analytic solution and the simulation

Table 1 Solar wind and photoelectrons parameters ( $\alpha$ is the elevation angle)

\begin{tabular}{cccc}
\hline & electrons & ions & photoelectrons \\
\hline Drifting Velocity, $\mathrm{km} / \mathrm{s}$ & 468 & 468 & - \\
Density, $\mathrm{cm}^{-3}$ & 8.7 & 8.7 & $64 \sin (\alpha)$ \\
Temperature, eV & 12 & 10 & 2 \\
\hline
\end{tabular}

with $f(\mathbf{x})=\left\{f_{1}(\mathbf{x}), f_{2}(\mathbf{x}), \ldots, f_{n}(\mathbf{x})\right\}$, where $n$ is the dimensionality of $\mathbf{x}$, the basis functions and $\beta=\left\{\beta_{1}, \beta_{2}, \ldots, \beta_{n}\right\}$ the vector of regression coefficients. In this paper, ordinary Kriging is selected which means that $F(\mathbf{x}, \beta) \equiv \beta$. All the following equations are based on ordinary Kriging. The covariance of $h(\mathbf{x})$ between two input points $\mathbf{x}$ and $\mathbf{w}$ is defined by

$$
\operatorname{cov}(h(\mathbf{x}), h(\mathbf{w}))=\sigma_{h}^{2} R_{\theta}(\mathbf{x}, \mathbf{w})
$$

where $\sigma_{h}^{2}$ is the random field variance and $R_{\theta}$ the correlation function defined by a set of parameters $\theta$. Several models exist to define the correlation function. The widely used anisotropic Gaussian model is given by

$$
R_{\theta}(\mathbf{x}, \mathbf{w})=\prod_{i=1}^{n} \exp \left[-\theta_{i}\left(x_{i}-w_{i}\right)^{2}\right]
$$

where $x_{i}$ and $w_{i}$ are the $i^{\text {th }}$ components of $\mathbf{x}$ and $\mathbf{w}$, respectively, and $\theta_{i}$ is a parameter. We use this model in this study.

Given any $\mathbf{x}$ in the input space, the Kriging prediction $G(\mathbf{x})$ follows a normal distribution $N\left(\mu(\mathbf{x}), \sigma^{2}(\mathbf{x})\right)$. The reason why the Kriging prediction is a random variable is that only a finite number of training samples are used to train the Kriging model. Therefore, there is epistemic uncertainty in the prediction and the uncertainty is modeled by the normal random variable. The larger is $\sigma(\mathbf{x})$, the more significant is the uncertainty. If $\sigma(\mathbf{x})=0$, the normal variable degenerates into a deterministic value $\mu(\mathbf{x})$, meaning that the prediction at this point is exact. Usually the prediction is only exact at training points because at training points, the exact output values are known to the Kriging model. Generally, $\mu(\mathbf{x})$ is used as the deterministic prediction. When $\sigma(\mathbf{x})$ is sufficiently small, $\mu(\mathbf{x})$ is an accurate prediction. On the contrary, when $\sigma(\mathbf{x})$ is large, $\mu(\mathbf{x})$ is not guaranteed to be an accurate prediction. In that case, more training samples are needed to update the Kriging model. With $\sigma(\mathbf{x})$ being capable to quantify the prediction accuracy,the Kriging model outweights many other metamodels. 


\section{B. Training Kriging Model for the Photoelectron Sheath Solution}

For a 1-D photoelectron sheath, the electric potential $\phi$ is a function of the vertical coordinate $z$ with parameters $\mathbf{p}=\left(v_{d}, T_{e}, T_{p}, n_{i, \infty}, n_{p}\right)$, where $v_{d}, T_{e}, T_{p}, n_{i, \infty}$, and $n_{p}$ represent solar wind drifting velocity, electron temperature, photoelectron temperature, density of ions at infinity, and density of photoelectrons, respectively.

Due to the aleatory uncertainty in lunar environment as well as the epistemic uncertainty, all components of $\mathbf{p}$ are modeled as interval variables, whose domain is $\Omega$. Because a closed-form expression of the solution to $\phi(z ; \mathbf{p}), \mathbf{p} \in$ $\Omega, z \in\left[Z_{\min }, Z_{\max }\right]$ is not available, obtaining the entire electric potential function $\phi(z ; \mathbf{p}), \mathbf{p} \in \Omega, z \in\left[Z_{\min }, Z_{\max }\right]$ is time-consuming. To obtain $\phi(z ; \mathbf{p})$ efficiently, employing a Kriging model is a good method. Some input-output sample pairs, i.e., training points, are used to train the Kriging model $\widetilde{\phi}(z ; \mathbf{p})$ for $\phi(z ; \mathbf{p})$. If the prediction uncertainty (or error) is too large, more training samples are added to update $\widetilde{\phi}(z ; \mathbf{p})$ until the prediction uncertainty is acceptable. Note that both $z$ and $\mathbf{p}$ are treated as input variables of $\widetilde{\phi}(z ; \mathbf{p})$, so the dimensionality of $\widetilde{\phi}(z ; \mathbf{p})$ is 6 .

To generate training samples, we first evenly discretize $p_{i}, i=1,2,3,4,5$ into $N_{i}, i=1,2,3,4,5$ points, respectively. Then $\Omega$ is accordingly discretized into $N_{\Omega}=\prod_{i=1}^{5} N_{i}$ points, denoted as $\mathbf{p}^{\mathrm{U}}$. The specific values of $N_{i}, i=1,2,3,4,5$ are determined by $\Omega$ as well as the required resolution. Since for any given $\mathbf{p} \in \Omega$, a numerical method, such as the Runge-Kutta integration method, is used to solve Poisson's equation to get a curve $\phi(z ; \mathbf{p}), z \in\left[Z_{\min }, Z_{\max }\right]$, we get a series of training samples along $z$-direction when $\mathbf{p}$ is fixed to a specific value. When we solve Poisson's equation using a numerical integration method, we also need to discretize $z$ into $N_{z}$ points, denoted as $\mathbf{z}^{\mathrm{U}}$. Then we evenly select $N_{\text {ini }}$ initial points of $\mathbf{p}$, denoted as $\mathbf{p}^{\text {ini }}$, from $\mathbf{p}^{\mathrm{U}}$. For each $\mathbf{p} \in \mathbf{p}^{\text {ini }}$, we get $N_{z}$ initial training samples from $\phi(z ; \mathbf{p})$ and hence currently we have $N_{z} N_{\text {ini }}$ initial training points. Since $\phi\left(Z_{\max } ; \mathbf{p}\right)$ is always 0 , for each $\mathbf{p} \in \mathbf{p}^{\mathrm{U}}$ we also have a training point $\left(Z_{\max }, \mathbf{p}, 0\right)$. As a result, there are in total $N_{z} N_{\text {ini }}+N_{\Omega}$ training points.

With the initial training points, we can build an initial Kriging model $\widetilde{\phi}(z ; \mathbf{p})$. In order to know if $\widetilde{\phi}(z ; \mathbf{p})$ is accurate enough, we need to come up with a metric to measure the overall accuracy. As mentioned in the previous subsection, $\sigma(z ; \mathbf{p})$ is able to measure the prediction error. However, $\sigma(z ; \mathbf{p})$ can only measure the prediction error at a specific point $(z ; \mathbf{p})$. To measure the overall accuracy, we use the metric $\Gamma$ which is given by

$$
\Gamma=\frac{1}{N_{z} N_{\Omega}} \sum_{i=1}^{N_{\Omega}} \sum_{j=1}^{N_{z}}\left(\left|\frac{\sigma\left(z^{(j)} ; p^{(i)}\right)}{\mu\left(z^{(j)} ; p^{(i)}\right)}\right|\right)
$$

where $z^{(j)}$ is the $j^{\text {th }}$ point in $\mathbf{z}^{\mathrm{U}}$, and $\mathbf{p}^{(i)}$ is the $i^{\text {th }}$ point in $\mathbf{p}^{\mathrm{U}}$. Actually, Eq. 23 is the mean absolute deviation coefficient of all prediction points. If $\Gamma<\gamma$, where $\gamma$ is a small positive number, the Kriging model $\widetilde{\phi}(z ; \mathbf{p})$ is said to be accurate. Otherwise, more training samples are necessary to update the Kriging model. To determine on which $\mathbf{p} \in \mathbf{p}^{\mathrm{U}}$ we should add training samples, we need to come up with a learning function [4]

$$
\mathbf{p}^{(\mathrm{next})}=\max _{\mathbf{p} \in \mathbf{p}^{\mathrm{U}}} \sum_{j=1}^{N_{z}}\left(\left|\frac{\sigma\left(z^{(j)} ; \mathbf{p}\right)}{\mu\left(z^{(j)} ; \mathbf{p}\right)}\right|\right)
$$

where $^{\text {(next) }}$ is the next training sample of $\mathbf{p}$. Then we solve Poisson's equation to get $N_{z}$ training points, fixing $\mathbf{p}$ to $\mathbf{p}^{\text {(next) }}$. Adding those training points to update $\widetilde{\phi}(z ; \mathbf{p})$, we get a higher prediction accuracy and hence a smaller value of $\Gamma$. Using similar procedures, we can update $\widetilde{\phi}(z ; \mathbf{p})$ adaptively until $\Gamma<\gamma$ is met. Obviously, $\gamma$ is a hyperparameter that controls the accuracy of the final $\widetilde{\phi}(z ; \mathbf{p})$. In general, the smaller is $\gamma$, the higher accuracy of $\widetilde{\phi}(z ; \mathbf{p})$ will we obtain.

\section{1-D Photoelectron Sheath: Kriging Modeling and UQ Analysis}

In this section, we present a study of 1-D photoelectron sheath including analytic solution (treated as a "black box" in the surrogate modeling step), Kriging model, and uncertainty quantification to demonstrate the approach described in Section IV

We consider the 1-D photoelectron sheath problem discussed in [5] as a test problem. The intervals of $v_{d}, T_{e}, T_{p}, n_{i, \infty}$, and $n_{p}$ are $468,000(1 \pm 0.1) \mathrm{m} / \mathrm{s}, 12(1 \pm 0.1) \mathrm{eV}, 2(1 \pm 0.1) \mathrm{eV}, 8.7(1 \pm 0.1) \mathrm{cm}^{-3}$, and $64(1 \pm 0.1) \mathrm{cm}^{-3}$, respectively, and then $\Omega=468,000(1 \pm 0.1) \mathrm{m} / \mathrm{s} \times 12(1 \pm 0.1) \mathrm{eV} \times 2(1 \pm 0.1) \mathrm{eV} \times 8.7(1 \pm 0.1) \mathrm{cm}^{-3} \times 64(1 \pm 0.1) \mathrm{cm}^{-3}$. We set $N_{i}=3, i=1,2,3,4,5$ and hence there are $N_{\Omega}=3^{5}=243$ points in $\mathbf{p}^{\mathrm{U}} .\left[Z_{\min }, Z_{\max }\right]=[0,60] \mathrm{m}$ is evenly discretized into $N_{z}=20$ points. In order to obtain the 243 electric potential profiles, we can solve Poisson's equations 243 times, each with a parameter combination from the 243 points. Figure 8 shows the 243 electric potential profiles. Each profile 
contains $N_{z}=20$ points, and hence there are in total $243 \times 20=4860$ points of data, which will be treated as the exact data points to verify corresponding data obtained by the proposed Kriging-based method.

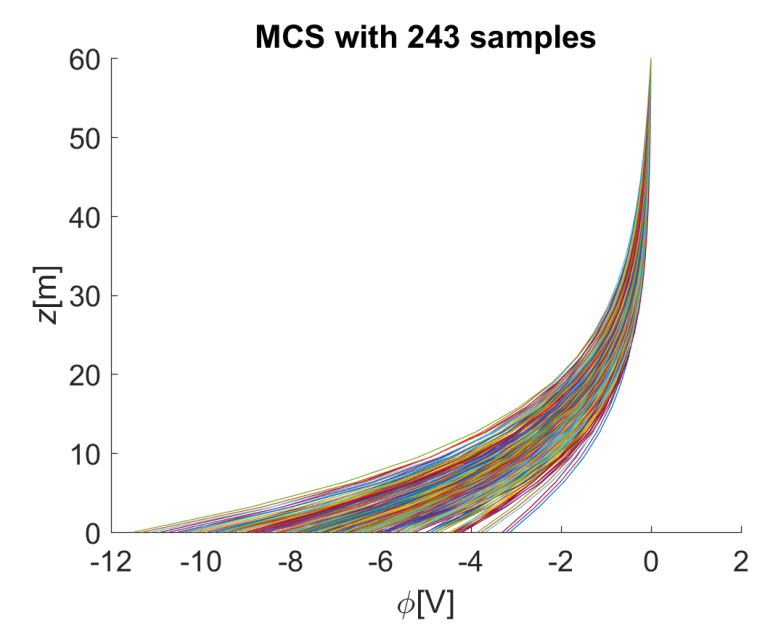

Fig. 8 Electric potential profiles obtained by MCS

To apply the proposed Kriging-based method, we first select 5 points $\mathbf{p}^{(i)}, i=1,2,3,4,5$ from $\mathbf{p}^{\mathrm{U}}$. Specific values of $\mathbf{p}^{(i)}, i=1,2,3,4,5$ are given in Table 2 At each of those 5 points, we solve Poisson's equation and get 5 electric potential profiles, which are shown in Fig. 9 (a). Since each profile contains $N_{z}=20$ points, currently there are in total 100 training points. As mentioned in Section IV] those points at $Z_{\max }$, where $\phi$ always takes 0 , are also used as training points. Therefore, there are in total $100+243=343$ training points for training the initial Kriging model. Figure 9 (b) shows corresponding 243 profiles estimated by the initial Kriging model. Obviously, the initial Kriging model is not accurate. After adding 10 more sample profiles to update the Kriging model, corresponding 243 profiles estimated by the Kriging model are shown in Fig. 9 (c). The accuracy is significantly improved. With $\gamma=0.05$, the proposed Kriging-based method converged after adding 28 sample profiles. The final electric potential profiles are shown in Fig. 9 (d). It shows that the final Kriging model is quite accurate. To obtain the upper and lower boundaries of the potential profiles, we evenly discretize $\Omega$ into 100,000 points, 100 points along each dimension, and then call the well-trained Kriging model to obtain the corresponding 100,000 potential profiles. With the 100,000 potential profiles, we finally obtain the upper and lower boundaries shown in Fig. 10.

Table 2 Initial training samples of $p$

\begin{tabular}{cccccc}
\hline \hline $\mathbf{p}^{(i)}$ & $v_{d}, \mathrm{~m} / \mathrm{s}$ & $T_{e}, \mathrm{eV}$ & $T_{p}, \mathrm{eV}$ & $n_{i, \infty}, \mathrm{cm}^{-3}$ & $n_{p}, \mathrm{~cm}^{-3}$ \\
\hline $\mathbf{p}^{(1)}$ & 421,200 & 10.8 & 1.8 & 7.83 & 57.6 \\
$\mathbf{p}^{(2)}$ & 421,200 & 12.0 & 2.2 & 8.7 & 64 \\
$\mathbf{p}^{(3)}$ & 468,000 & 10.8 & 2.0 & 9.57 & 70.4 \\
$\mathbf{p}^{(4)}$ & 468,000 & 13.2 & 2.0 & 8.7 & 57.6 \\
$\mathbf{p}^{(5)}$ & 514,800 & 12.0 & 1.8 & 9.57 & 64 \\
\hline \hline
\end{tabular}




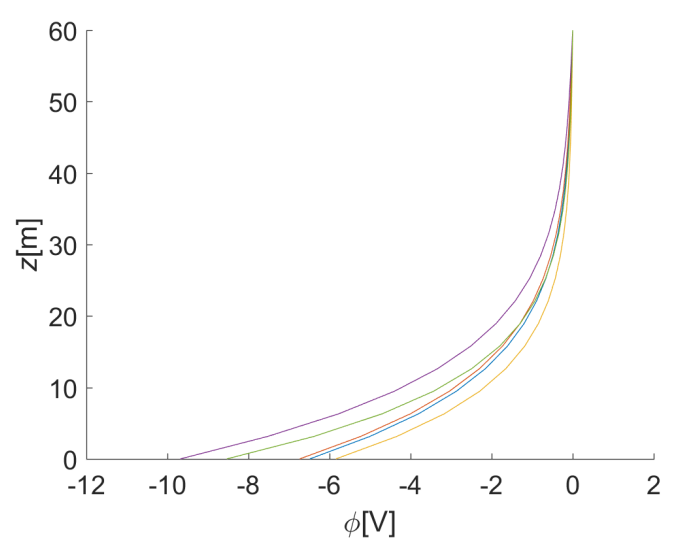

(a) The initial training samples

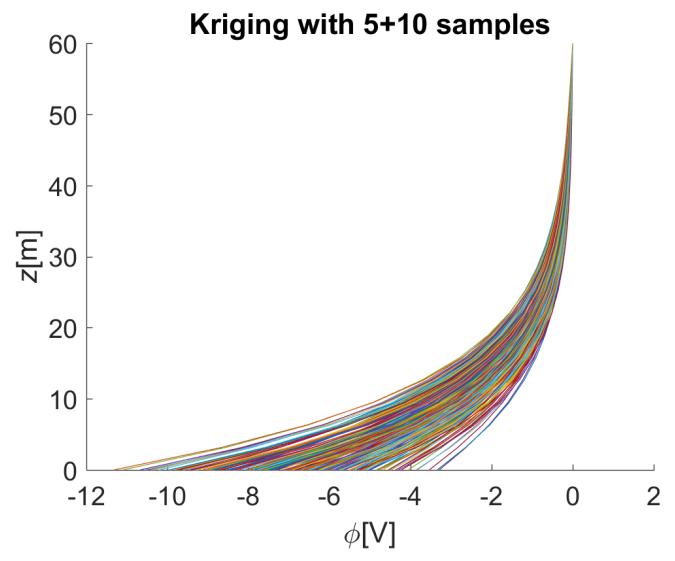

(c) The updated Kriging model

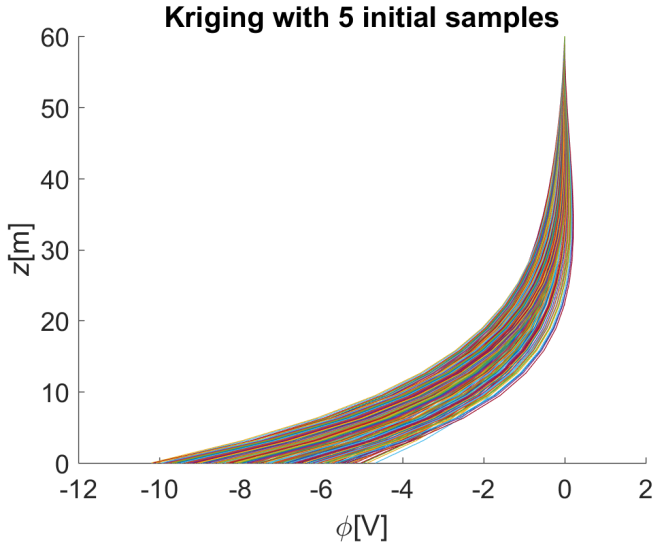

(b) The initial Kriging model

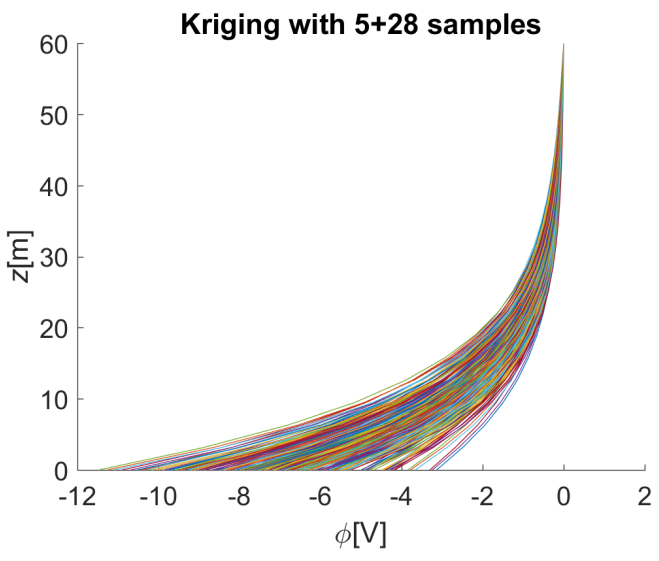

(d) The final Kriging model

Fig. 9 Electric potential profiles estimated by the Kriging model

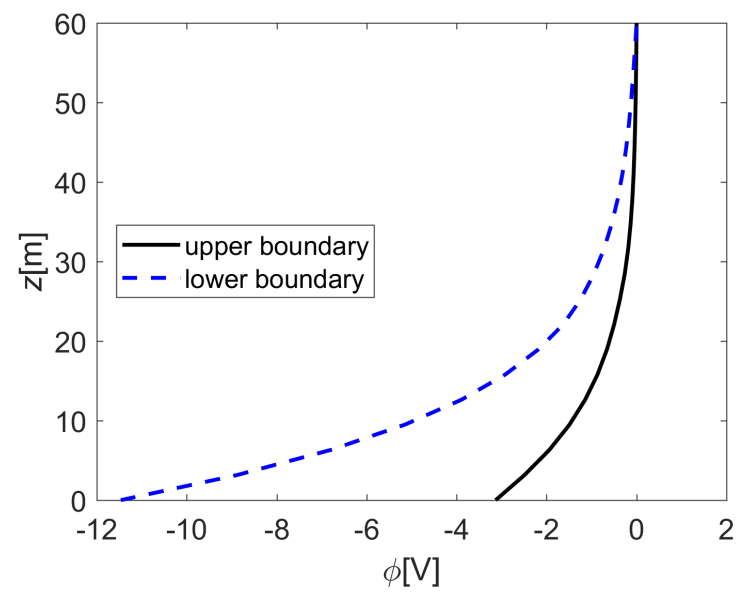

Fig. 10 Potential profile boundaries obtained by Kriging model 


\section{Conclusion}

We introduced the analytic approach to obtain the 1-D electric potential profile, which is a function of the distance from the lunar surface in this study. The shape of the potential profile will be affected by the sun elevation angle, there are three different types of potential profiles in the assumption, according to the different elevation angle. As the elevation angle increases, the potential profile will transform from Type $\mathrm{C}$ to Type $\mathrm{A}$, and then to Type $\mathrm{B}$. However, even though both Type A and Type B can be obtained from the analytic solution, there should be only one stable profile in reality.

We also used a FD-PIC code to run a numerical simulation, and compare the result with the analytic solution. We ran the simulation with different elevation angles, and the comparison shows that the result obtained from the simulation matches the analytic solution very well, which demonstrates the validity of the code.

Then the FD-PIC code and the analytic solution have been used as the black box to support the uncertainty quantification analysis. We used the Kriging model to predict the 1-D photoelectron sheath, and the result shows the accuracy of the Kriging model, which demonstrates the ability and the accuracy of the UQ analysis in this research.

Ongoing work is focused on 2-D and 3-D configurations of the lunar surface to perform UQ analysis.

\section{Acknowledgments}

We would like to thank Prof. Joseph Wang of the University of Southern California for many helpful discussions. This work is financially supported in part by NASA-Missouri Space Grant Consortium through NASA-EPSCoR Missouri, and NSF-CMMI \# 1923799. J. Zhao would also thank the Missouri S\&T Graduate Studies Travel Fund for the travel support to AIAA SciTech 2020.

\section{References}

[1] Fu, J. H. M., "Surface potential of a photoemitting plate," Journal of Geophysical Research (1896-1977), Vol. 76, No. 10, 1971, pp. 2506-2509. doi:10.1029/JA076i010p02506, URL https://agupubs.onlinelibrary.wiley.com/doi/abs/10. 1029/JA076i010p02506

[2] Nitter, T., Havnes, O., and Melands $\varnothing$, F., "Levitation and dynamics of charged dust in the photoelectron sheath above surfaces in space," Journal of Geophysical Research: Space Physics, Vol. 103, No. A4, 1998, pp. 6605-6620. doi:10.1029/97JA03523, URL http://onlinelibrary.wiley.com/doi/10.1029/97JA03523/abstract

[3] Jones, D. R., Schonlau, M., and Welch, W. J., "Efficient global optimization of expensive black-box functions," Journal of Global optimization, Vol. 13, No. 4, 1998, pp. 455-492.

[4] Echard, B., Gayton, N., and Lemaire, M., "AK-MCS: an active learning reliability method combining Kriging and Monte Carlo simulation," Structural Safety, Vol. 33, No. 2, 2011, pp. 145-154.

[5] Jeong, H., and Wang, J., "Fully Kinetic Modeling of Photoelectron Sheath in Drifting Plasma," 39th Plasmadynamics and Lasers Conference, Seattle, Washington, 2008. doi:10.2514/6.2008-3784, URL http://arc . aiaa.org/doi/abs/10.2514/6.20083784 